\section{Colaborative learning environment (cle) en la asignatura de Fundamentos de gestión empresarial con la aplicación de $m$-learning y assessment feedback/ feedforward mediante pares y sistemas de autorización presencial y virtual}

\author{
SONIA MARTÍN-GÓMEZ ${ }^{a}$ \\ CRISTINA MASA-LORENZO ${ }^{b}$
}

\begin{abstract}
RESUMEN Los cambios originados hace unos años con el Proceso de Bolonia y el establecimiento del Espacio Europeo de Educación Superior (EEES) para lograr la convergencia y la comparabilidad en los sistemas educativos requieren introducir modificaciones en el proceso de aprendizaje tradicional. En este estudio se analiza cómo introducir esos cambios en la asignatura básica de Fundamentos de gestión empresarial, la cual se imparte en las facultades de Ciencias Económicas y Empresariales de nuestras universidades, aplicado al caso de la Universidad San Pablo-CEU. Para esto, se propone la creación de entornos colaborativos de aprendizaje (collaborative learning environment-CLE), con la utilización para su desarrollo del aprendizaje colaborativo basado en problemas, mediante el uso de redes sociales a través de dispositivos móviles distintos ( $m$-learning), y con la realización de feedback a fin de mejorar el aprendizaje entre los propios estudiantes y con el profesor a través de tutorías, bien sean presenciales o bien virtuales. Se trata de crear un entorno que incluya todos aquellos recursos que ayudan al alumno a buscar y organizar información y conocimiento. Estos recursos, que se pueden compartir con grupos de trabajo, en nuestro caso serán fundamentalmente redes sociales, dispositivos móviles y la plataforma virtual de la universidad a través de la cuál puede gestionarse y llevarse a cabo el proceso de formación. El curso funciona en dicha plataforma como un entorno exclusivo que contiene, entre otros medios, un sistema de mensajería interna y otro de foros, diseñados con el propósito de llevar a cabo tutorías individuales y colectivas.
\end{abstract}

PALABRAS CLAVE aprendizaje móvil, entornos colaborativos de aprendizaje, evaluación, redes sociales, retroalimentación, tutoría.

\section{HISTORIA DEL ARTÍCULO}

¿CÓMO CITAR?:

Martín-Gómez, S. \& Masa-Lorenzo, C. (2018). Colaborative learning environment (cle) en la asignatura de Fundamentos de gestión empresarial con la aplicación de m-learning y assessment feedback/ feedforward mediante pares y sistemas de autorización presencial y virtual. Perspectiva Empresarial, 5(1), 17-30. http://dx.doi. org/10.16967/rpe.v5n1a2

RECIBIDO: 31 de octubre de 2017 APROBADO: 10 de enero de 2018 CORRESPONDENCIA: Sonia Martín-Gómez, Calle Julián Romea, 23, 28003 Madrid, España.

a Doctora, Responsable de la unidad docente fundamentos de empresa de la Universidad de San Pablo-CEU, España. Correo electrónico: margom@ceu.es.

b Doctora, Secretaria Académica Departamento de Economía de la Empresa de la Universidad San Pablo-CEU -CEU, ESpaña. Correo electrónico: cmasalor@ceu.es 


\section{¿CóMO CITO EL ARTÍCULO? HOW TO CITE THIS PAPER?}

CHICAGO:

Martín-Gómez, Sonia y

Masa-Lorenzo, Cristina.

2018. "Colaborative learning environment (cle) en la asignatura de Fundamentos de gestión empresarial con la aplicación de m-learning y assessment feedback/ feedforward mediante pares y sistemas de autorización presencial y virtual". Perspectiva Empresarial 5(1): 17-30. http://dx.doi. org/10.16967/rpe.v5n1a2

MLA:

Martín-Gómez, Sonia y MasaLorenzo, Cristina. "Colaborative learning environment (cle) en la asignatura de Fundamentos de gestión empresarial con la aplicación de m-learning y assessment feedback/ feedforward mediante pares y sistemas de autorización presencial y virtual". Perspectiva Empresarial 5.1 (2018): 17-30. Digital. http:// dx.doi.org/10.16967/rpe. v5n1a2

\section{Collaborative Learning Environment (CLE) in the Subject of Fundamentals of Business Management Applying M-Learning and Assessment Feedback/ Feedforward through Peers and Physical and Virtual Authorization Systems}

ABSTRACT The changes originated a few years ago by the Bologna Process and the establishment of the European Higher Education Area (EHEA) to achieve convergence and comparability in education systems require that modifications be introduced in the traditional learning process. This study analyzes how to introduce these changes in the basic subject of Fundamentals of Business Management, which is taught in the School of Economic and Business Sciences, applied to the case of the Universidad San Pablo-CEU. For this, the creation of collaborative learning environments (CLE) is proposed using problem-based collaborative learning through social media on different mobile devices (m-learning) and providing feedback to improve learning among students themselves and with the professor through tutorials, either face-to-face or virtual. It is about creating an environment that includes all those resources that help the student to find and organize information and knowledge. In our case, these resources, which can be shared with work groups, will be mainly social media, mobile devices, and the university's virtual platform through which the training process can be managed and carried out. The course works on this platform as an exclusive environment that contains, among other means, internal messaging and forum systems designed for giving individual and collective tutorials.

KEYWORDS mobile learning, collaborative learning environments, assessment, social media, feedback, tutoring.

\section{Colaborative learning environment (CLE) na matéria de Fundamentos de} gestão empresarial com a aplicação de $m$-learning e assessment feedback/ feedforward mediante pares e sistemas de autorização presencial e virtual

RESUMO As mudanças que foram originadas há alguns anos com o Processo de Bolonha e o estabelecimento do Espaço Europeu de Educação Superior (EEES) para alcançar a convergência e a comparabilidade nos sistemas educativos requerem introduzir modificações no processo de aprendizagem tradicional. Neste estudo foi analisado como introduzir essas mudanças no programa básico de Fundamentos de Gestão Empresarial, que é dado nas faculdades de Ciências Econômicas e Empresariais, aplicado ao caso da Universidad San Pablo-CEU. Para isso, propõe-se a criação de ambientes colaborativos de aprendizagem (collaborative learning environment - CLE) destinados ao desenvolvimento de uma aprendizagem colaborativa baseada em problemas, mediante o uso de redes sociais através de dispositivos móveis diferentes ( $m$-learning), e com a realização de feedback, a fim de melhorar a aprendizagem, entre os próprios estudantes e o professor, através de tutorias, sejam elas presenciais ou virtuais. Trata-se de criar um entorno que inclua todos os recursos que ajudam o aluno a buscar e organizar informação e conhecimento. Esses recursos, que podem ser compartilhados com grupos de trabalho, no nosso caso serão, fundamentalmente, redes sociais, dispositivos móveis e a plataforma virtual da universidade, através da qual é possível administrar e levar a cabo o processo de formação. 0 curso funciona na plataforma mencionada, como um ambiente exclusivo que contém, entre outros meios, um sistema de mensagem interna e outro de fóruns, desenhados com o propósito de levar a cabo tutorias individuais e coletivas.

PALAVRAS CHAVE aprendizagem móvel, ambientes colaborativos de aprendizagem, avaliação, redes sociais, feedback, tutoria. 


\section{Marco y justificación teórica}

Como consecuencia de la implantación definitiva de la Declaración de Bolonia se requiere una implicación mayor del alumno en el proceso de enseñanza-aprendizaje, de forma que no solo se preocupe por intentar asimilar todo aquello que se le transmite, sino que además deberá mostrar cómo reconstruye todos esos conocimientos a fin de conformar su aprendizaje personal, de qué formas es capaz de elaborar su propia red de contenidos y cómo puede utilizarla para enfrentarse a problemas de su campo disciplinar. Asimismo, se incrementa la consideración de la necesidad de aprender a trabajar de forma colaborativa con otras personas.

Esta novedosa implicación del alumno requiere invertir la clase con el objetivo de liberar tiempo, lo que se conoce como sistema flipped classroom, el cual consiste en modificar la forma en que los contenidos se entregan y así dar mayor tiempo a la práctica y a la aplicación de teorías y conceptos (Talbert, 2012). Los estudiantes revisan los contenidos fuera del aula y la práctica es realizada durante el transcurso de la clase.

Se trata de una metodología pedagógica que propone un modelo de aprendizaje caracterizado por el rol activo del aprendiz, lo cual permitirá desarrollar un aprendizaje colaborativo y fomentar el coaprendizaje.

Entre los diferentes sistemas de motivación del alumno para el aprendizaje y de organización de la docencia por parte del profesor, al parecer el que aporta mejores resultados a los alumnos es el sistema cooperativo, pues en el sistema individualista cada alumno trabaja para conseguir su meta -al margen de los compañeros-, sin que esto influya en la consecución de sus propias metas a cada uno de los demás. En el sistema competitivo cada alumno trabaja de manera independiente a fin de alcanzar su meta, consciente de que ello supone que los demás compañeros no han de alcanzar la suya. Cada estudiante se propone, entonces, como finalidad, quedar mejor con relación a los demás en aquellos aspectos que se tienen en cuenta en la evaluación.

Por el contrario, en el sistema cooperativo cada alumno alcanza la meta que se ha propuesto en la medida en que los compañeros de su grupo alcanzan las suyas. La interacción con los compañeros es fundamental en razón a los beneficios que obtienen en la elaboración y construcción del conocimiento.
En consecuencia, será preciso plantear cambios en el proceso de enseñanza-aprendizaje tradicional y sustituirlo por otro tipo de enseñanza en el que se destaquen las estrategias de aprendizaje participativo y colaborativo, para lo cual resulta de utilidad aplicar las tecnologías de la información y la comunicación (TIC) a la creación de los denominados "entornos colaborativos de aprendizaje" o CLE, los cuales deben favorecer la adquisición de las competencias que se demandan en la asignatura.

Estas actividades formativas coordinadas o colaborativas - a las que también hace referencia la Agencia Nacional de Evaluación de la Calidad (Aneca) - se pueden conseguir al incluir en los CLE el uso de dispositivos móviles y de plataformas virtuales, es decir, al incorporar las nuevas tecnologías al aula.

El aprendizaje colaborativo es el empleo didáctico de grupos pequeños en el que los alumnos trabajan juntos con el objetivo de obtener los mejores resultados de aprendizaje tanto a nivel individual como grupal. Se trata, por tanto, de aplicar metodologías de aprendizaje que incentivan la colaboración entre los alumnos con el propósito de conocer, compartir y ampliar la información que cada uno tiene sobre un tema, y compartir datos mediante espacios de discusión reales o virtuales.

Dentro de estos grupos colaborativos se pueden emplear distintas metodologías de trabajo. Dadas las características del programa de la asignatura de Fundamentos de gestión empresarial, en la que se busca poner en práctica este sistema de aprendizaje, optaremos por la técnica jigsaw en su versión más sencilla, y la puzzle para determinados aspectos del programa, según analizaremos posteriormente en la descripción del proyecto. Así, se reparten entre los alumnos casos o problemas de empresas próximos a la realidad sobre los que trabajarán de forma colaborativa.

El aprendizaje colaborativo basado en problemas (ACBP) comienza su presencia sistemática en la docencia universitaria a mediados de la década de los sesenta del siglo pasado en la Facultad de Medicina de la Universidad de McMaster, en Canadá.

De acuerdo con Cabero (2003), este aprendizaje colaborativo se define como una metodología de enseñanza basada en la creencia de que el aprendizaje se incrementa cuando los estudiantes desarrollan destrezas cooperativas para aprender 
y solucionar los problemas y acciones educativas en los cuales se ven inmersos.

A lo largo del proceso de trabajo grupal los alumnos deben adquirir responsabilidad y confianza en el trabajo realizado en el grupo, y desarrollar así la habilidad de dar y recibir críticas orientadas a la mejora de su desempeño y del proceso de trabajo del grupo, lo que hace, por tanto, indispensable la existencia de una retroalimentación a distintos niveles (feedback).

El conocimiento sobre las actividades del resto de los participantes, la comunicación en tiempo real y diferido por varios canales, la coordinación de actividades junto con la facilidad en el diseño de actividades y el registro del proceso de aprendizaje a través de la conservación del historial de actividades de la secuencia del intercambio de información y de las producciones individuales y grupales en un continuo, hacen necesario desarrollar entornos colaborativos de aprendizaje, presenciales o virtuales que se apoyen en redes sociales y plataformas educativas.

En el ámbito del aprendizaje de las personas (Attwell, 2007; Downes, 2010), se entiende un entorno de aprendizaje como una idea pedagógica, como una práctica de las personas para aprender valiéndose de la tecnología, es decir, como una forma de ver el aprendizaje con Internet, sus relaciones, su dinámica y su naturaleza.

Por ello, se concibe un personal learning environment (PLE) como el conjunto de herramientas, fuentes de información, conexiones y actividades que cada persona utiliza de forma asidua con el propósito de aprender.

Entre estas herramientas, el aprendizaje móvil -también denominado "m-learning" - ofrece métodos modernos de apoyo al proceso de aprendizaje mediante el uso de instrumentos móviles, tales como los ordenadores portátiles y las tabletas informáticas, los lectores MP3, los teléfonos inteligentes (smartphones) y los teléfonos móviles.

Actualmente, el uso de estas nuevas tecnologías y la aplicación de las redes sociales a la docencia pueden favorecer un desarrollo óptimo de este aprendizaje colaborativo. Así lo trasmitía en el 2006 el documento elaborado por la Comisión para la Renovación de las Metodologías Educativas en la Universidad, realizado por el Ministerio de Educación y Ciencia, en el que se plantea el proceso de construcción del Espacio Europeo de Educación Superior (EEES) como:
La oportunidad perfecta para impulsar una reforma que no debe quedarse en una mera reconversión de la estructura y contenidos de los estudios, sino que debe alcanzar al meollo de la actividad universitaria, que radica en la interacción profesores-estudiantes para la generación del aprendizaje. (Consejo de Coordinación Universitaria, 2006, pp. 7)

También la Comisión Europea en el 2007 habla de las competencias digitales como una de las ocho competencias clave que todas las personas van a precisar para estar en capacidad de desarrollarse personal y profesionalmente; por tanto, es fundamental generar procesos educativos que favorezcan la adquisición de la competencia digital por parte de los estudiantes universitarios.

Prácticamente, la totalidad de los países europeos fomentan la utilización de una gran variedad de herramientas TIC en el proceso de enseñanza-aprendizaje, pero son relativamente pocos los países que recomiendan o sugieren el uso de dispositivos móviles o de libros electrónicos. Es en este punto que nuestro proyecto busca ser pionero, ya que son muchos los autores que justifican el uso de las redes sociales en las experiencias docentes y la formación del profesorado (Cabero \& Marín, 2014).

Se trata de que el alumno perciba la importancia de aplicar sus conocimientos en el uso de redes sociales en sus dispositivos móviles a sus enseñanzas universitarias, ya que la actitud negativa mostrada por los alumnos hacia una tecnología o forma de utilización repercute sobre el tipo de interacciones que establecen y los rendimientos que alcanzan con esta (Hung \& Cheng, 2013).

A esto se debe añadir que la importancia de nuevas tecnologías y redes sociales es cada vez mayor, pues al referirse exclusivamente a nuestro país, según datos del "Informe Ditrentria sobre Mobile en España y en el mundo", un 57 \% de la población visita redes sociales a través del smarthphone, frente a un $15 \%$ que lo hace a través de la tableta.

Entre las redes sociales, según el "Estudio anual de redes sociales 2017", realizado por la Interactive Adverstising Bureau Spain (IAB Spain) - la Asociación de la publicidad, el Marketing y la Comunicación Digital en España-, Facebook es aún la red social por excelencia, al ser la más visitada (91\%), seguida de WhatsApp, YouTube y Twitter, con un uso semanal de algo más de tres horas. 
Dado lo anterior, nos planteamos su uso como herramienta de aprendizaje en un sistema de aula invertida y aprendizaje colaborativo para compartir diversos aspectos de la asignatura: temas teóricos, casos prácticos, artículos y foros de discusiones sobre estos, indicaciones sobre resoluciones de casos, videos, etc. Se trata de que el alumno perciba la importancia de aplicar sus conocimientos en el uso de redes sociales en sus dispositivos móviles a sus enseñanzas universitarias.

Estos cambios en la cultura del aprendizaje implican también cambios en los sistemas de evaluación, de ahí que se tienda a una evaluación orientada al aprendizaje universitario (leaning-oriented assessment). Dentro de esta evaluación, orientada a aprender, aparecen los conceptos de feedback (retroalimentación) y feedforward (proalimentación), como instrumentos que permiten mejorar los resultados del aprendizaje de forma periódica, presencial o virtual.

El concepto de evaluación mediante feedback (assessment feedback) ha ido transformándose a lo largo del tiempo, en muchas ocasiones como consecuencia de cambios en el propio concepto de evaluación. No existe un consenso generalizado sobre qué se entiende por evaluación en términos académicos y muy pocas investigaciones sobre la evaluación mediante feedback, mientras que algunos autores defienden el feedback como un tipo de instrumento de medida y, para otros, forma parte integral de la evaluación (Clark, 2011).

Esta conceptualización, como parte de un proceso continuo de apoyo al aprendizaje tanto en el contexto inmediato como en futuras adquisiciones de aprendizaje, se conoce como feedforward o "pasos a seguir para mejorar a partir de ahora"; algunos autores creen que tiene mayor poder para estimular el aprendizaje que el feedback (Knight, 2006).

Por otra parte, es necesario que los estudiantes desarrollen la capacidad de evaluar su propio trabajo por sí mismos, es decir, practiquen un sistema de evaluación de 180 grados en el que, además del docente, ellos mismos valoren su trabajo. La evaluación orientada al aprendizaje debe hacer uso de las técnicas de autoevaluación y de evaluación por compañeros, ya que sirven para implicar a los estudiantes en la evaluación mediante el desarrollo de las capacidades necesarias para ello (Carless, Joughin \& Liu, 2007).

Actualmente, los alumnos se quejan de la forma en que se utiliza el feedback como sistema de evaluación, pues no debemos olvidar que "todavía quedan muchas cosas que no se conocen sobre cómo diseñar mejor los sistemas de evaluación que conducen a un mejor aprendizaje para estudiantes de educación superior" (Sadler, 2010).

En nuestro caso, para que el feedback sea más efectivo nos vamos a apoyar en rúbricas diseñadas tanto para la evaluación de los profesores como de los propios alumnos, y nos apoyaremos en tutorías no solo presenciales sino también virtuales (E-assesment feedback-EAF), a fin de que la retroalimentación se divulgue de forma más óptima.

Se trata de involucrar en la retroalimentación diferentes medios electrónicos para que el gap entre la realización de una determinada actividad y su feedback sea pequeño, pero también es necesario realizar un esfuerzo a fin de que la brecha entre recibir y actuar sea escasa, ya que las percepciones individuales de la retroalimentación por parte de los alumnos pueden ser muy distintas, de modo que reaccionen en algunos casos de forma inmediata y requieran, en otros, de una explicación adicional (la cual se propone proporcionar mediante tutorías académicas).

La rúbrica es un instrumento de evaluación basado en una escala cuantitativa o cualitativa asociada a unos criterios preestablecidos que miden las acciones del alumnado sobre los aspectos de la tarea o la actividad que se evaluarán. Es, por tanto, un instrumento de evaluación del desempeño de los estudiantes que se desarrolla en el desglose de sus componentes a fin de conseguir la calificación total.

La retroalimentación se puede concebir y percibir de forma muy distinta por el estudiante, lo que obliga a tener que incidir en esta en las tutorías académicas. Weaver (2006) señala los niveles de madurez intelectual de los estudiantes y las experiencias vividas como factores que afectan la receptividad de la retroalimentación.

La consecución del cambio de perspectiva didáctica en el proceso de enseñanza-aprendizaje que exige la convergencia europea debería acompañarse de un cambio de perspectiva en el proceso tutorial, de manera que se logre integrar ambos procesos en una misma práctica y en relación con las necesidades de aprendizaje del estudiante que, en nuestro caso, pasan por realizar un feedback adecuado.

Para esto sería conveniente poner en práctica la tutoría integrada, en la que además de que el docente sea el guía del proceso de aprendizaje, pueda realizar retroalimentación individual o en 
pequeños grupos a los alumnos, dentro del aula o fuera de ella y de forma presencial o virtual.

En este sentido, la Universidad CEU-San Pablo siempre ha sido pionera en las tutorías académicas y ha realizado un esfuerzo por que se conviertan en su factor de diferenciación.

\section{Descripción del estudio planteado}

\section{Población}

Fundamentos de gestión empresarial es una asignatura que se imparte en el primer semestre del primer curso del Grado en Administración y Dirección de Empresas (GAD), del Grado en Marketing (GMK), y del Grado en Economía (GNF), así como en los Grados Simultáneos de Economía y GAD, Economía y Periodismo, y GAD y Marketing de la Facultad de Ciencias Económicas y Empresariales. De igual forma, en el primer curso del Grado Simultáneo de Derecho y GAD de la Facultad de Derecho, de Marketing y Publicidad de la Facultad de Humanidades y Ciencias de la Comunicación, y en los Grados Simultáneos de Farmacia y GAD en la Facultad de Farmacia, e Ingeniería de Sistemas y GAD de la Escuela Politécnica Superior de la Universidad San Pablo-cEu.

En todos los casos tiene asignada una carga de seis créditos ECTS. Se trata de una asignatura introductoria en el caso de los alumnos del Grado de Administración y Dirección de Empresas y del Grado en Marketing, así como en los Grados Simultáneos de la Facultad de Derecho, Farmacia y la Escuela Politécnica Superior, ya que en los siguientes cursos profundizarán en las distintas áreas que conforman la empresa. Sin embargo, en el caso del Grado en Economía constituye una visión global de lo que supone una empresa y su gestión.

Por esta razón, aunque sea una asignatura introductoria, es necesario que los estudiantes consigan una visión global de lo que representa una empresa, las distintas subfunciones que la integran y su gestión.

A lo largo de la asignatura se pretende que el alumno sea capaz de entender en qué consiste la gestión funcional de una empresa. Para esto se deben identificar cuáles son las principales actividades o áreas que constituyen una empresa y cómo se interrelacionan.

\section{Objetivos}

Se planteó como objetivo principal crear entornos colaborativos de aprendizaje en la asignatura de Fundamentos de gestión empresarial, y apostar así por el uso de una metodología dialógica, es decir, una metodología activa basada en el diálogo razonado y reflexivo por parte del alumno, apoyado en valores de compromiso y solidaridad con los demás compañeros.

Como objetivos secundarios de apoyo a esta metodología se plantearon los siguientes:

- Favorecer el aprendizaje con el uso de dispositivos móviles (m-learning) que permitan afianzar los entornos de aprendizaje mediante redes sociales.

- Modificar los sistemas tradicionales de evaluación al apostar por un sistema de retroalimentación y mejora (assessment feedback/ feedforward) mediante pares, esto es, generado por los propios alumnos, y por sistemas de tutorización del profesor de forma presencial y virtual. Todo lo anterior apoyado en rúbricas en las que quede constancia de los puntos fuertes y débiles que se han tenido en cuenta para generar la evaluación del alumno.

- Convertir el trabajo en equipo en el eje en torno al cual ha de girar una propuesta metodológica de esta índole, ya que el trabajo en grupo favorece la comunicación más abierta, la asunción de roles y de compromisos dentro y fuera del equipo, la cooperación y la solidaridad.

\section{Características básicas}

Permite alcanzar mejoras en tres ámbitos: en el propio estudiante, en el grupo de trabajo, en la evaluación y mejoras académicas. La tabla 1 resume las más importantes.

\section{Metodología de enseñanza-aprendizaje}

En la enseñanza de la asignatura Fundamentos de gestión empresarial se utilizan actualmente, en 
TABLA 1. Ventajas de los entornos colaborativos de aprendizaje.

\begin{tabular}{|c|c|c|c|}
\hline ESTUDIANTE & GRUPO DE TRABAJO & EVALUACIÓN & MEJORAS ACADÉMICAS \\
\hline $\begin{array}{l}\text { - Tolerancia y respeto. } \\
\text { - Creatividad. } \\
\text { - Comunicación entre } \\
\text { iguales. } \\
\text { - Pensamiento crítico y } \\
\text { reflexivo. } \\
\text { - Compromiso y } \\
\text { responsabilidad. } \\
\text { - Aprendizaje de los demás. } \\
\text { - Solidaridad para compartir } \\
\text { conocimiento. }\end{array}$ & $\begin{array}{l}\text { - Conocimiento personal } \\
\text { y profesional de los } \\
\text { demás. } \\
\text { - Establecer } \\
\text { planificaciones y } \\
\text { agendas. } \\
\text { - Compromiso de } \\
\text { retroalimentación } \\
\text { grupal. } \\
\text { - Guiar la discusión } \\
\text { grupal a objetivos. }\end{array}$ & $\begin{array}{l}\text { - Feedback de } \\
\text { aprendizaje. } \\
\text { - Feedback de relación } \\
\text { con el equipo. } \\
\text { - Feedback con tutor. } \\
\text { - Uso de rúbricas. }\end{array}$ & $\begin{array}{l}\text { - Visión global de la materia en un } \\
\text { caso práctico. } \\
\text { - División por áreas simulando una } \\
\text { empresa. } \\
\text { - Uso del material de la blackboard y } \\
\text { páginas web. } \\
\text { - Proyecto abierto, con varias } \\
\text { soluciones y posibles debates. } \\
\text { - Prueba final acorde con este caso. } \\
\text { - Desarrollo de competencias básicas } \\
\text { y específicas }\end{array}$ \\
\hline
\end{tabular}

Nota. Elaboración propia.

la mayoría de las universidades españolas, las siguientes estrategias docentes:

- Clases teóricas. En las que se expone el contenido teórico de la asignatura mediante los recursos audiovisuales apropiados en clases magistrales y seminarios.

- Clases prácticas. Dedicadas a la resolución de ejercicios con la utilización, por lo general, de técnicas grupales (aprendizaje cooperativo). Los resultados de los distintos ejercicios se analizan en clase tras su resolución y puesta en común.

- Tutorías. Se trata de orientar y aclarar al alumno aquellos aspectos concretos que no se han llegado a entender en el aula de forma grupal.

A estas técnicas ya utilizadas queremos sumar la metodología de flipped classroom mediante el aprendizaje colaborativo basado en proyectos apoyado en entornos virtuales (redes sociales) y bajo el enfoque del mobile learning.

Los aspectos que resumen la eficacia del aprendizaje colaborativo en nuestro proyecto docente para la asignatura de Fundamentos de gestión empresarial se detallan a continuación.

La idea del proyecto se expone en los primeros días de clase, cuando se explican sus líneas principales, cómo se califica y el cronograma a seguir, y se les solicita a los estudiantes que formen grupos de cinco alumnos; se les asignan los roles que van a asumir cada uno siguiendo la técnica jigsaw, en la cual dentro de cada grupo se decide la parte del proyecto a resolver por cada uno de sus integrantes, por lo que cada uno de ellos tiene una única tarea inicial y esta es necesaria a fin de resolver el problema en su conjunto.
Una vez resuelta su parte del trabajo cada alumno volverá al grupo y presentará un informe de su labor al resto de integrantes, en el que explica y aclara los conceptos más complejos y destaca las principales conclusiones y decisiones que se van a tomar.

Para un mejor desarrollo de cada una de las partes se recomienda, antes de la presentación del informe al grupo, la reunión de los alumnos por temas específicos (con o sin tutor).

Esta puesta en común, la cual puede ser informal y ayuda a una mejor resolución, es lo que denominamos "reuniones de expertos". Con la unión de los distintos informes quedaría resuelto el problema inicial.

El uso de esta técnica en nuestra asignatura, resumida en diez pasos, se desarrolla de la siguiente forma: 1. Dividir el aula en grupos de cinco o seis personas; 2 . Nombrar un líder o portavoz del grupo; 3. Dividir el programa de la asignatura entre cinco y seis partes; 4. Asignar a cada estudiante una de las partes; 5. Elaboración por parte de cada alumno de su informe correspondiente; 6. Reunión de "expertos" (previa a la reunión del grupo) que mejorarán los informes individuales; 7. Reunión de los grupos de trabajo; 8. Exposición de cada una de las partes por el "experto" correspondiente; 9. Exposición del trabajo final (normalmente a través del "líder"); y 10. Evaluación de cada uno de los grupos.

En nuestro caso, al tener en cuenta la asignatura Fundamentos de gestión empresarial, los roles o partes en las que se divide el proyecto se corresponden con los temas principales del programa que aparece en la guía docente:

- Director financiero y de administración de la empresa. Sería el líder del grupo y el 
responsable final de todo el proyecto de forma conjunta, lo que supone tanto las inversiones como la captación de financiación.

- Responsable de compras y almacenes de materias primas y productos acabados. También se encarga de la localización óptima de las instalaciones.

- Responsable de producción. Incluye la planificación, su programación y el control de proyectos actuales y futuros.

- Responsable de la función comercial. Incluye fijación de precios, distribución, publicidad, etc., y el resto de aspectos relacionados con el marketing de la empresa.

- Responsable de recursos humanos. Se ocupa de todos los aspectos de la dirección de personas y del resto de factores dispositivos.

Con el fin de facilitar a los alumnos esta tarea se genera en el aula una tormenta de ideas en la que se debate, dentro de los distintos grupos espontáneos que se hayan formado, la asignación de las diferentes actividades y se elige al que va a ser el líder-director financiero de la empresa.

Después de un corto espacio temporal que permita a los estudiantes analizar las conclusiones a las que han llegado, se solicita el envío de un correo electrónico al profesor en el que se registre a los responsables de las distintas áreas y quién asumirá la figura de director.

Una vez organizados formalmente los grupos de trabajo, el profesor-tutor repartirá a los distintos líderes los casos de las empresas cuyas operaciones se deben analizar.

Los problemas planteados y sus posibles soluciones se entregarán al profesor de acuerdo con el cronograma establecido al efecto, y al final del semestre el director de cada grupo expondrá las gestiones definitivas realizadas en la empresa, lo cual se denominará "Sesión final".

En esta sesión se admite la participación de alumnos de otros grupos que opinen sobre las conclusiones del grupo expositor y las acciones que han decidido tomar. Igualmente, el profesor podrá preguntar cualquier duda y solicitar al alumno su justificación cuantitativa en la pizarra en caso necesario.

El papel del profesor es importante en este tipo de metodología pues debe fomentar la cooperación dentro de los grupos y delegar parte de su autoridad en los distintos líderes de los grupos, de modo que los alumnos sean los verdaderos protagonistas del proyecto.

El profesor deberá ayudar a los alumnos en la resolución de dudas, guiarlos y valorar sus iniciativas. Una parte muy importante del éxito del proyecto depende, por tanto, del papel que desempeña el docente como tutor, tanto a nivel presencial como virtual. Por esto, es importante que los profesores tutores sigan unas pautas en las primeras tutorías.

Pensamos que en la primera sesión de tutoría es apropiado emplear algún recurso que conduzca al conocimiento mutuo, y generar así pequeños diálogos sobre experiencias de aprendizaje anteriores y su grado de conformidad, y sobre aspectos que desearía mejorar en sí mismo o en el nuevo grupo que recién comienza a trabajar.

De esta forma, el tutor comienza a conocer a los individuos y propicia el diseño de estrategias grupales, así como el plan de acción a trabajar en el plano individual, lo que permite ayudarlos a que mejoren los aspectos que ellos han detectado y les gustaría mejorar, de modo que se acelere el "buen hacer" del equipo.

Un segundo aspecto que debe formar parte de esa primera sesión de tutoría es establecer las reglas de comportamiento dentro y fuera dela aula que serán respetadas por los integrantes del grupo, y crear así una comunidad de aprendizaje que cuente con normas por escrito en una especie de código ético de esa comunidad o grupo.

Un tercer aspecto a aclarar es que, independientemente de que el resultado de la actividad deba presentarse en clase, es conveniente que el grupo informe con brevedad al resto de la clase acerca de cómo han trabajado y qué aspectos del trabajo en equipo han funcionado bien, así como de las dificultades que han encontrado, de modo que puedan resolverse para trabajos en grupo en el futuro (Slavin, 1990).

Finalmente, también se debe hablar en la primera tutoría sobre la interdependencia de los miembros del equipo y la responsabilidad individual. La interdependencia implica que cada estudiante es responsable del resultado final y, al mismo tiempo, depende de los demás integrantes del equipo para alcanzar el resultado.

\section{Sistema de evaluación}

En nuestro estudio, las rúbricas se emplearán como instrumentos de medición en los que se 
establecen criterios por niveles mediante la disposición de escalas que permiten determinar la calidad de la ejecución de los estudiantes en unas tareas específicas.

Por tanto, se hace necesaria la elaboración, por parte de toda la unidad docente, de un cuadro de doble entrada o matriz de evaluación en el cual se expresen de forma explícita en el eje vertical (filas) los aspectos que se evaluarán e informarán de la calidad de la tarea, y en el eje horizontal (columnas) los cuantificadores (10, 9, 8, etc.), así como las escalas o calificativos (excelente, bien, regular, malo) que se asignarán a los diferentes niveles de logro.

Las escalas pueden construirse en una gran variedad de formas y niveles de complejidad, pero todas deben contar con algunos elementos indispensables, como, por ejemplo, enfocarse en medir los objetivos planteados (contenidos, procedimientos y actitudes); además, deben utilizar un rango que permita medir el desempeño, y contar con los criterios específicos ordenados en niveles, de forma que indiquen en qué grado se cumple lo planeado.

En nuestro caso, la evaluación comienza desde las propias tutorías que el profesor realiza periódicamente en grupo, de forma presencial o virtual (a través de Facebook, Blackboard, correo electrónico, etc.). De esta forma, el tutor evalúa el proceso del grupo y de sus miembros al recoger las observaciones pertinentes, las cuales comentará en caso necesario con los alumnos. Para esto, se realizará una planificación previa de las tutorías (según aparece en el "Plan de trabajo" de este documento), y se construirá una ficha de seguimiento similar en la que quedará evidenciada la tutoría.

En cuanto al trabajo del grupo (exposiciones e informes sobre el problema), el profesor-tutor lo calificará tras la defensa, y si es posible otros grupos de alumnos podrán dar opiniones sobre estos. Esta calificación del profesor oscilará entre 0 y 50 (al tratarse de grupos formados por cinco alumnos), para lo cual también se seguirá una plantilla o rúbrica.

La calificación obtenida por el grupo se repartirá entre todos los miembros de este, de la forma que el equipo estime más justa y objetiva. Así, entonces, se evalúan las competencias de cada estudiante con la utilización de varias fuentes (observadores): el profesor y otros compañeros, de acuerdo con un sistema de evaluación de 180 grados. La justificación del uso de este sistema de valoración es doble: por un lado, como docentes conocemos a nuestros alumnos en función de su comportamiento en clase, en tutoría y del resultado final de su trabajo; sin embargo, por otra, la rutina diaria en cuanto a formas y modos de trabajo y actuación la conocen mejor sus propios compañeros, por lo que pueden aportar valoraciones importantes que se deben tener en cuenta en el momento de calificar un proyecto.

Este reparto de la calificación también se realiza con ayuda de una rúbrica que favorece la reflexión y la equidad, al exigir una justificación de la nota asignada a cada miembro por parte de los demás compañeros.

Todos los resultados relativos a la evaluación del proyecto y la información necesaria para mostrar cómo se han ido desarrollando los distintos trabajos se recopilan en una carpeta o portafolio denominada "carpeta del proceso de aprendizaje".

Un resumen de este portafolio se hará público por parte del profesor en la plataforma virtual usada por la universidad, de tal forma que el estudiante conozca de forma clara los resultados del aprendizaje desarrollado durante el semestre.

\section{Recursos necesarios}

El material básico de la asignatura se encuentra en una plataforma educativa a disposición de todos los alumnos matriculados en la asignatura; todos los cálculos que se derivan de la información facilitada y que permiten resolver los casos planteados se pueden realizar mediante hoja de cálculo Microsoft Excel, mientras que para la exposición del trabajo se contará con el apoyo de los recursos informáticos disponibles en las distintas aulas.

Además, los alumnos pueden usar las redes sociales a fin de favorecer su aprendizaje, por lo que previamente a la implantación de esta nueva metodología de enseñanza se ha buscado investigar sobre cómo perciben los estudiantes universitarios las redes sociales. Al centrarnos en Facebook - por ser la red más utilizada en cuanto al tiempo que le dedican medido en horas-semana y sus usos-, se podrá establecer si es útil como recurso didáctico en nuestro estudio. La conclusión obtenida es que su uso se restringe al ámbito no académico y su temporalidad es escasa, aunque es preciso resaltar que, encuestados aquellos alumnos que usan Facebook con fines académicos, un porcentaje muy elevado lo utiliza con el fin de interactuar con miembros del grupo de trabajo 
e intercambiar información de clase sobre ejercicios o casos a presentar.

Por tanto, la principal mejora que propone este proyecto son unos cambios necesarios en la relación entre el profesor y el alumno, lo que potencia el papel de este último en su proceso de aprendizaje. Al contar con el apoyo de Facebook y el mobile learning, al conectarse a esta red social desde cualquier dispositivo se puedan conseguir las siguientes utilidades: 1 . Entorno de colaboración para el grupo de trabajo; 2 . Medio de comunicación: profesor- alumno, alumno-profesor, alumno-alumno y alumno-experto profesional; 3. Plataforma virtual de aprendizaje; 4. Entorno de debate y discusión (a través del foro de discusión grupal de Facebook); 5. Realización de tutorías individuales o grupales: en las individuales el alumno puede plantear dudas o problemas con su trabajo en particular, mientras que en las grupales el profesor puede reunirse de forma virtual con los directores de las distintas áreas de operaciones o con los líderes (representantes) de cada grupo, con el fin de analizar el grado de avance del caso planteado.

En relación con el grupo, el tutor deberá plantearse la consecución de los siguientes aspectos:

- Crear un ambiente de confianza y respeto en el aula.

- Fomentar y estimular la discusión grupal, de forma que nadie sea un mero espectador del proceso.

- Establecer ese vínculo necesario entre la nueva información planteada en el problema y sus conocimientos previos.

- Ayudar a los alumnos a que se atrevan a pensar, a tomar riesgos y a ser capaces de adelantar una hipótesis y luego probar su validez.

- Ayudar a resumir y sintetizar la información que ha sido discutida y así obtener una visión de conjunto de todos los datos analizados con el uso de, por ejemplo, mapas conceptuales.

- Elaborar al final un registro del progreso del grupo que luego transmitirá a sus alumnos.

\section{Resultados esperables}

No disponemos de evidencias sobre posibles resultados a alcanzar con este proyecto de innovación docente, de modo que el éxito o fracaso del presente proyecto depende de la evaluación de los resultados que se obtendrán en la asignatura en los distintos grados en los que se imparte durante los próximos cursos académicos.

Los resultados del proyecto se van a medir en un futuro a través de indicadores relacionados con el rendimiento académico de los estudiantes, como lo son la tasa de éxito (porcentaje de estudiantes que aprueban la asignatura en relación con los que se presentan al examen), tasa de rendimiento (en relación con los que se han matriculado en la asignatura) y tasa de expectativa (relación entre presentados y matriculados).

Al no disponer todavía de los resultados derivados del presente trabajo, hemos calculado los indicadores de rendimiento académico a partir de los datos correspondientes a la convocatoria ordinaria de los seis cursos académicos anteriores, con la finalidad de poderlos contrastar en un futuro con los datos nuevos.

Estos indicadores arrojan unos resultados aceptables, ya que tanto la tasa de éxito como la de rendimiento están en torno al 75-80 \%, con una evolución creciente desde el curso 11-12; no obstante, se encuentra aproximadamente un $20 \%$ por debajo de la tasa de expectativa, y es este diferencial el que se pretende reducir con la nueva metodología basada en proyectos.

\section{Autoevaluación del estudio}

La autoevaluación se obtendrá a partir del diseño y la aplicación de un cuestionario que permitirá realizar una evaluación coherente y objetiva del nuevo sistema de aprendizaje que se propone, así como su evaluación con rúbricas.

Este cuestionario se remitirá a los alumnos la última semana del curso sin realizar una selección de los participantes, utilizando como único criterio la voluntad de cumplimentar el cuestionario de valoración de forma objetiva.

El cuestionario, que utiliza una escala tipo Likert de cinco niveles ( 1 = total desacuerdo; $-5=$ total acuerdo), se configura en tres dimensiones que corresponden a habilidades adquiridas, metodología de aprendizaje basada en proyectos, y una dimensión adicional sobre el grado de satisfacción general que el estudiante manifiesta sobre este nuevo sistema. El número de enunciados preguntas según la dimensión es de ocho para las habilidades adquiridas, nueve para la metodología del ACBP, y tres sobre satisfacción general, es decir, un total de 20 enunciados a valorar por el estudiante. 
Un ejemplo de este cuestionario se presenta en la tabla 2.

También serán elementos de autoevaluación los resultados obtenidos en cuanto a tasa de rendimiento, éxito y expectativa en la asignatura y su comparativa entre distintos cursos académicos, así como las conclusiones obtenidas por los docentes de la asignatura y las puestas en común en las distintas reuniones de coordinación.

TABLA 2. Cuestionario de evaluación para el alumnado sobre las nuevas metodologías empleadas

\section{CUESTIONARIO DE VALORACIÓN METODOLOGÍA APLICADA}

GRADO:

CURSO:

HABILIDADES ADQUIRIDAS

$\begin{array}{lllll}1 & 2 & 3 & 4 & 5\end{array}$

1. COMUNICACIÓN: mayor capacidad de comunicación verbal y mejor uso del lenguaje técnico.

2. SÍNTESIS: mejor capacidad de resumir para acoplarse a tiempos de exposición.

3. CRÍTICA: mejora en las aportaciones de puntos de vista distintos sobre bases teóricas.

4. AUTONOMÍA: el grupo es capaz de resolver problemas sin la ayuda directa del profesor.

5. INICIATIVA: el grupo realiza planteamientos y genera soluciones de forma independiente, fuera de lo meramente solicitado.

6. IMPLICACIÓN: los alumnos son capaces de aportar sugerencias, resoluciones, etc.

7. CREATIVIDAD: Son capaces de idear distintos recursos tanto didácticos como visuales para mejorar la exposición.

8. PLANIFICACIÓN: He aprendido a gestionar mejor los tiempos.

METODOLOGÍA ABPC

9. RECURSOS: los materiales de apoyo son útiles en el propósito de solucionar el problema planteado.

10. OBJETIVOS: los alumnos conocen en todo momento la finalidad del proyecto.

11. CONTENIDOS: el problema planteado nos ha ayudado/incentivado a ampliar los contenidos de la asignatura.

12. CARGA DE TRABAJO: la resolución del problema no ha supuesto un incremento de las horas de dedicación a la asignatura.

13. INTERÉS: esta metodología ha mejorado mi interés por la asignatura y mi visión de esta.

14. SISTEMA DE EVALUACIÓN DEL PROYECTO: la forma de evaluación ha sido la adecuada.

15. RELACIONES CON COMPAÑEROS: este sistema ha mejorado las relaciones interpersonales con los compañeros de la clase.

16. RELACIONES CON PROFESORES: este sistema ha mejorado las relaciones interpersonales con los profesores de la asignatura.

17. En general, considero que este método de trabajo es mejor que el tradicional.

\section{SATISFACCIÓN GENERAL}

18. La asignatura es importante para mi desarrollo profesional.

19. He comprendido la asignatura a nivel práctico.

20. Me gusta esta asignatura.

Nota. El 1 se corresponde con la calificación más baja y el 5 con la más alta. Elaboración propia. 


\section{Plan de trabajo de la metodología propuesta}

El cronograma de actividades, las evaluaciones y las tutorías relacionadas con el ACBP aparecen en la tabla 3 por meses y semanas, y se han destacado en color gris $\square$ las actividades relacionadas con el ACBP.

La aplicación del método de aprendizaje basado en proyectos colaborativos apoyado en Facebook, así como su uso en dispositivos móviles en la asignatura Fundamentos de gestión empresarial resulta útil, ya que para muchos alumnos la aplicación de esta asignatura en la práctica es compleja por distintos motivos, tal y como se ha puesto de manifiesto con anterioridad.

A nivel internacional, se considera que es necesario diseñar experiencias de aprendizaje activo diferentes a la clase magistral, los cuales consigan que el alumno entienda mejor los conceptos de gestión de operaciones y su importancia estratégica en la empresa, se adapten a las necesidades curriculares y cumplan con los requisitos que demandan las empresas (Fish, 2008; Piercy, 2010).

Por otra parte, diversas investigaciones confirman que el método docente tiene una clara influencia en variables como, por ejemplo, la motivación, la comprensión, la percepción del aprendizaje y la dificultad percibida en la materia
(Alfalla-Luque, Medina-López \& Arenas-Márquez, 2011). Es decir, aunque la materia en sí misma pueda afectar a estas variables, la forma en que se imparte ejerce una importante influencia (MartínPeña, Díaz-Garrido \& Flores, 2012). Esto significa que a través de la metodología docente utilizada se puede modificar la actitud del estudiante y su percepción con respecto a la materia.

Finalmente, pensamos que la experiencia específica de esta asignatura arrojará interesantes conclusiones que podrán generalizarse a otras impartidas en la Facultad, de modo que se favorezca el poner este nuevo método de enseñanza en marcha en los próximos cursos si los resultados alcanzados en la asignatura piloto son los esperados.

Además, nuestro trabajo tiene claras implicaciones académicas y prácticas, en el sentido de que ofrecemos un ejemplo de una forma de introducir el método de enseñanza cooperativo en cualquier otra asignatura que deba ser adaptada a los criterios exigidos por el EEES.

\section{Conclusiones}

La innovación en docencia es la base para la mejora de la formación y de la capacitación que obtengan los alumnos, los futuros empresarios

TABLA 3. Cronograma de actividades

\begin{tabular}{|c|c|c|c|c|}
\hline & $1^{\mathrm{a}}$ semana & $2^{a}$ semana & $3^{\mathrm{a}}$ semana & $4^{\mathrm{a}}$ semana \\
\hline Septiembre & & $\begin{array}{l}\text { Presentación } \\
\text { asignatura y guía } \\
\text { docente (1h) } \\
\text { Explicación del ABP (1h) }\end{array}$ & $\begin{array}{l}\text { Brainstorming para } \\
\text { formación de grupos } \\
\text { y elección de empresa } \\
\text { (1h) } \\
\text { Seminarios }\end{array}$ & Seminarios \\
\hline Octubre & $\begin{array}{l}\text { Seminarios } \\
\text { Primera tutoría grupal } \\
\text { con responsables } \\
\text { financieros }\end{array}$ & Seminarios & $\begin{array}{l}\text { Seminarios } \\
\text { Segunda tutoría grupal } \\
\text { con responsables de } \\
\text { compras y almacén }\end{array}$ & Seminarios \\
\hline Noviembre & $\begin{array}{l}\text { Seminarios Tercera } \\
\text { tutoría grupal con } \\
\text { responsables de } \\
\text { producción }\end{array}$ & Seminarios & $\begin{array}{l}\text { Seminarios } \\
\text { Cuarta tutoría grupal } \\
\text { con responsables de } \\
\text { comercialización. }\end{array}$ & Seminarios \\
\hline Diciembre & $\begin{array}{l}\text { Seminarios } \\
\text { Quinta tutoría grupal } \\
\text { con directores-líderes }\end{array}$ & $\begin{array}{l}\text { Casos globales }(2 \mathrm{~h}) \\
\text { Exposiciones finales de } \\
\text { proyectos }\end{array}$ & $\begin{array}{l}\text { Evaluaciones de } \\
\text { proyectos (profesor) } \\
\text { Cuestionarios } \\
\text { Evaluaciones de } \\
\text { proyectos (alumnos) }\end{array}$ & $\begin{array}{l}\text { Publicación final } \\
\text { calificaciones }\end{array}$ \\
\hline
\end{tabular}


y los directivos. Se persigue la adquisición de conocimientos teóricos y prácticos, así como el desarrollo de la capacidad del alumnado para abordar esquemas generales de resolución de problemas, pero se debe enfatizar mucho más en el desarrollo de sus competencias a fin de acceder al conocimiento, tanto de forma individual como cooperativa.

En consecuencia, la metodología del proceso de enseñanza-aprendizaje-evaluación debe girar en torno a estas competencias previamente establecidas, y el profesor deberá preocuparse por que el método o métodos que utilice a la hora de desarrollar su actividad conlleven a una participación activa del estudiante en la construcción de su propio aprendizaje.

Se plantea restringir de manera significativa las clases teórico-expositivas para la transmisión de contenidos, renovar los métodos de aprendizaje hacia otros más participativos apoyados en el uso de redes sociales y dispositivos móviles, y cambiar los recursos para la evaluación de los estudiantes con la introducción de otros más adecuados y diferentes a los exámenes, al contemplar una evaluación formativa que permita al estudiante conocer sus propios progresos a lo largo de todo su proceso de aprendizaje y al otorgar a la evaluación un carácter sumativo, en busca de fórmulas que contribuyan a apoyar unos aprendizajes en otros y así proporcionen coherencia a todo el conjunto.

El aprendizaje requiere cinco condiciones básicas: 1. La motivación, el interés por trabajar y aprender sobre el tema; 2. La comprensión de la temática; 3. La participación activa en el proceso de la enseñanza-aprendizaje; 4. La aplicación de lo visto en clase a situaciones o actividades del quehacer empresarial, de modo que los aprendizajes se puedan contrastar con la realidad; 5 . El aprendizaje debe ser colaborativo, es decir, en equipo, ya que el trabajo en grupo implica una interacción entre iguales que se pueden apoyar en el proceso de aprendizaje.

Cuando un estudiante trabaja en equipo de forma colaborativa para la consecución de un fin común, adquiere una serie de habilidades sociales, responsabilidad individual y demás habilidades inherentes a la dinámica del grupo, lo que le permite alcanzar con éxito las competencias fijadas en la asignatura, así como tener más éxito en su futura inserción laboral y poner en práctica una serie de valores innatos, tales como la solidaridad y el compromiso hacia los demás, lo cuales se encuentran al alza en la sociedad en general y son iconos de nuestra Institución.

Las metodologías activas y la dinámica de grupos presentan ventajas académicas, pero no debemos olvidar que también permiten la satisfacción de otras necesidades personales y sociales de los alumnos. La colaboración que supone el trabajo en grupos pequeños favorece la vivencia de experiencias en las relaciones interpersonales que favorecen el desarrollo de las tareas comunes y facilita el aprendizaje, la asunción de la responsabilidad en el propio trabajo personal y en el trabajo del grupo, así como el fortalecimiento de la personalidad de los alumnos.

La tendencia actual al aprendizaje directo y personalizado proseguirá y se reforzará en los próximos años en todas las universidades con el apoyo de los dispositivos móviles y las redes sociales (m-learning). La tecnología móvil puede dar apoyo a los educandos en su exploración del mundo circundante y en el desarrollo de sus propias soluciones a problemas complejos mientras trabajan en colaboración con sus pares y bajo la guía de profesores competentes.

Este cambio en el proceso de aprendizaje debe ir acompañado de una modificación sustancial en la evaluación. La evaluación debe permitir, a partir de la retroalimentación actual y futura (feedback) feedforward), entender la evolución de los procesos formativos para, a partir de esa información, orientar y dinamizar propuestas de avance. La retroalimentación proporciona oportunidades para alcanzar las expectativas y permite modificar la dificultad de las metas y obtener reconocimiento, por lo tanto, es importante proporcionar oportunidades de evaluación a los estudiantes a fin de determinar cómo se están desempeñando.

Además, si esta evaluación forma parte del proceso de enseñanza-aprendizaje, requiere la presencia también del alumno - de modo corresponsable - en la configuración del proceso evaluativo. Una forma de facilitar esta labor a profesores y alumnos es mediante la confección de rúbricas de valoración.

Como conclusión final debemos recordar que en nuestra calidad de docentes nuestra tarea culmina solo si nuestros alumnos realmente han aprendido, y no necesariamente porque nosotros enseñamos. Se hace imprescindible poner en marcha metodologías que lleven al alumnado a interactuar entre ellos, a tener experiencias cuasi-reales y, así, al cuestionarse la realidad empresarial, hagan lo posible por modificarla en aquellos 
aspectos mejorables: "La esperanza es desear que algo suceda. La fe es creer que va a suceder. La valentía es hacer que suceda". (Anónimo, s. f.)

\section{REFERENCIAS}

Alfalla-Luque, R., Medina-López, C. \& Arenas-Márquez, F. (2011). Mejorando la formación en dirección de operaciones: la visión del estudiante y su respuesta ante diferentes metodologías docentes. Cuadernos de Economía y Dirección de la Empresa, $14(2), 40-52$.

Attwell, G. (2007). The personal learning environments-the future of eLearning? eLearning Papers, 2(1), 1-7.

Cabero, J. (2003). Principios pedagógicos, psicológicos y sociológicos del trabajo colaborativo: su proyección en la tele-enseñanza. En F. Martínez, (Coord.), Redes de comunicación en la enseñanza (129-156), Barcelona: Paidós.

Cabero, J. \& Marín, V. (2014). Posibilidades educativas de las redes sociales y el trabajo en grupo. Percepciones de los alumnos universitarios. Revista Comunicar, 42(XXI), 165-172.

Carless, D., Joughin, G. \& Liu, N. F., (2007). How assessment supports learning: learning-oriented assessment in action. Hong Kong: Hong Kong University Press.

Clark, I. (2011). Formative assessment: policy, perspectives, and practice. Florida Journal of Educational Administration \& Policy, 4, 158-180.

Consejo de Coordinación Universitaria (2006). Propuesta para la renovación de las metodologías universitarias. Madrid: Ministerio de Educación y Ciencia.
Downes, S. (2010). Learning Networks and Connective Know -ledge. In H. Yang, \& S. Yuen (Eds.). Collective Intelligence and ELearning 20 Implications of Web Based Communities and Networking. (pp. 1-26) Hershey, PA: IGI Global.

Fish, L. (2008). Graduate student project: employer operations management analysis. Journal of Education for Business, 84(1), 18-30.

Hung, S-H., \& Cheng, M. J. (2013). Are you ready for knowledge sharing? An empirical study of virtual communities. Computers \& Education, (62), 8-17.

Knight, P. (2006). The local practices of assessment, Assessment y Evaluation in Higher Education, 31(4), 435-452.

Martín-Peña, M. L., Díaz-Garrido, E. \& Flores, S. (2012). Operations management in services: an analysis of competence and methodologies from the perspective of student (EHEA). Working Papers on Operations Management, 5(2), 1-14.

Piercy, N. (2010). Experiential learning: the case of the production game. Decision Sciences Journal of Innovative Education, 8(1), 275-280.

Sadler, R. (2010). Beyond feedback: developing student capability in complex appraisal. Assessment \& Evaluation in Higher Education, 35(5), 535-550.

Slavin, R. E. (1990). Cooperative learning. Theory, research and practice. Needham Heights, MA: Allyn and Bacon.

Talbert, R. (2012). Inverted classroom. Colleagues, 9(1), Artículo 7.

Weaver, M. R. (2006). Do students value feedback? Student perceptions of tutors' written responses. Assessment and Evaluation in Higher Education, 31, 379-394. 